\title{
ASYMPTOTIC ISOPERIMETRY OF BALLS IN METRIC MEASURE SPACES
}

\author{
Romain TESSERA
}

\begin{abstract}
In this paper, we study the asymptotic behavior of the volume of spheres in metric measure spaces. We first introduce a general setting adapted to the study of asymptotic isoperimetry in a general class of metric measure spaces. Let $\mathcal{A}$ be a family of subsets of a metric measure space $(X, d, \mu)$, with finite, unbounded volume. For $t>0$, we define

$$
I_{\mathcal{A}}^{\downarrow}(t)=\inf _{A \in \mathcal{A}, \mu(A) \geq t} \mu(\partial A) .
$$

We say that $\mathcal{A}$ is asymptotically isoperimetric if $\forall t>0$

$$
I_{\mathcal{A}}^{\downarrow}(t) \leq C I(C t),
$$

where $I$ is the profile of $X$. We show that there exist graphs with uniform polynomial growth whose balls are not asymptotically isoperimetric and we discuss the stability of related properties under quasi-isometries. Finally, we study the asymptotically isoperimetric properties of connected subsets in a metric measure space. In particular, we build graphs with uniform polynomial growth whose connected subsets are not asymptotically isoperimetric.
\end{abstract}

\section{Introduction}

The study of large scale isoperimetry on metric measure spaces has proven to be a fundamental tool in various fields ranging from geometric group theory $[\mathbf{6}],[\mathbf{1 0}]$ to analysis and probabilities on graphs and manifolds $[\mathbf{1}],[\mathbf{2}]$. One of the targets of this paper is to find a simple setting adapted to the large scale study of isoperimetric properties. This includes some general assumptions on metric measure spaces, a convenient notion of "large scale" boundary of a subset, and a family of maps preserving the large scale isoperimetric properties. There are two

2000 Mathematics Subject Classification. Primary: 53C23; Secondary: 05C99. Key words. Isoperimetry, large-scale geometry, balls, metric measure spaces. 
kinds of questions concerning isoperimetry [11]: what is the isoperimetric profile? What are the subsets that optimize the isoperimetric profile? Here, we will formulate similar questions in a large scale setting: we will not be interested in the exact values of the isoperimetric profile but in its asymptotic behavior and we will consider sequences of subsets that optimize "asymptotically" the isoperimetric profile. Dealing with general metric measure spaces, the family of balls seems to be a natural candidate for optimizing asymptotically the isoperimetric profile. Nevertheless, we will see that even under apparently strong assumptions on the space $X$, this is not always the case. Let us be more precise.

\subsection{Boundary of a subset and isoperimetric profile.}

Let $(X, d, \mu)$ be a metric measure space. Let us denote $B(x, r)$ the closed ball of center $x$ and radius $r$. We suppose that the measure $\mu$ is Borel, supported on $X$ and $\sigma$-finite. For any measurable subset $A$ of $X$, any $h>0$, write

$$
A_{h}=\{x \in X, d(x, A) \leq h\}
$$

and

$$
\partial_{h} A=A_{h} \cap\left(A^{c}\right)_{h} .
$$

Let us call $\partial_{h} A$ the $h$-boundary of $A$, and $\partial_{h} B(x, r)$ the $h$-sphere of center $x$ and radius $r$.

Definition 1.1. Let us call the $h$-profile the nondecreasing function defined on $\mathbf{R}_{+}$by

$$
I_{h}(t)=\inf _{\mu(A) \geq t} \mu\left(\partial_{h} A\right),
$$

where $A$ ranges over all $\mu$-measurable subsets of $X$ with finite measure.

This definition of large-scale boundary has the following advantage: under some weak properties on the metric measure space $X$, we will see in Section 3.1 that in some sense, the boundary of a subset $A \subset X$ has a thickness "uniformly comparable to $h$ ". This will be play a crucial role in the proof of the invariance of "asymptotic isoperimetric properties" under large-scale equivalence (see Section 1.3).

\subsection{Lower/upper profile restricted to a family of subsets.}

Let $(X, d, \mu)$ be a metric measure space. In order to study isoperimetric properties of a family of (measurable) subsets of $X$ with finite, unbounded volumes, it is useful to introduce the following notions 
Definition 1.2. Let $\mathcal{A}$ be a family of subsets of $X$ with finite, unbounded volumes. We call lower (resp. upper) $h$-profile restricted to $\mathcal{A}$ the nondecreasing function $I_{h, \mathcal{A}}^{\downarrow}$ defined by

$$
I_{h, \mathcal{A}}^{\downarrow}(t)=\inf _{\mu(A) \geq t, A \in \mathcal{A}} \mu\left(\partial_{h} A\right)
$$

(resp. $\left.I_{h, \mathcal{A}}^{\uparrow}(t)=\sup _{\mu(A) \leq t, A \in \mathcal{A}} \mu\left(\partial_{h} A\right)\right)$.

Definition 1.3. Consider two monotone functions $f$ and $g: \mathbf{R}_{+} \rightarrow \mathbf{R}_{+}$. Say that $f \approx g$ if there exist some constants $C_{i}$ such that $C_{1} f\left(C_{2} t\right) \leq$ $g(t) \leq C_{3} f\left(C_{4} t\right)$ for all $t \in \mathbf{R}_{+}$.

The asymptotic behavior of a monotone function $\mathbf{R}_{+} \rightarrow \mathbf{R}_{+}$may be defined as its equivalence class modulo $\approx$.

We get a natural order relation on the set of equivalence classes modulo $\approx$ of monotone functions defined on $\mathbf{R}_{+}$by setting

$$
(f \preceq g) \Leftrightarrow\left(\exists C_{1}, C_{2}>0, \forall t>0, f(t) \leq C_{1} g\left(C_{2} t\right)\right) .
$$

We say that the family $\mathcal{A}$ is asymptotically isoperimetric (resp. strongly asymptotically isoperimetric) if for all $A \in \mathcal{A}$

$$
I_{h, \mathcal{A}}^{\downarrow} \preceq I_{h}
$$

(resp. $I_{h, \mathcal{A}}^{\uparrow} \preceq I_{h}$ ).

Remark 1.4. Note that asymptotically isoperimetric means that for any $t$ we can always choose an optimal set among those of $\mathcal{A}$ whose measure is larger than $t$ whereas strongly asymptotically isoperimetric means that every set of $\mathcal{A}$ is optimal (but the family $(\mu(A))_{A \in \mathcal{A}}$ may be lacunar). In almost all cases we will consider, the family $(\mu(A))_{A \in \mathcal{A}}$ will not be lacunar, and strong asymptotic isoperimetry will imply asymptotic isoperimetry.

\subsection{Large scale study.}

Let us recall the definition of a quasi-isometry (which is also sometimes called rough isometry).

Definition 1.5. Let $(X, d)$ and $\left(X^{\prime}, d^{\prime}\right)$ be two metric spaces. One says that $X$ and $X^{\prime}$ are quasi-isometric if there is a function $f$ from $X$ to $X^{\prime}$ with the following properties.

(a) There exists $C_{1}>0$ such that $[f(X)]_{C_{1}}=X^{\prime}$.

(b) There exists $C_{2} \geq 1$ such that, for all $x, y \in X$,

$$
C_{2}^{-1} d(x, y)-C_{2} \leq d^{\prime}(f(x), f(y)) \leq C_{2} d(x, y)+C_{2} .
$$


Example 1.6. Let $G$ be a finitely generated group and let $S_{1}$ and $S_{2}$ two finite symmetric generating sets of $G$. Then it is very simple to see that the identity map $G \rightarrow G$ induces a quasi-isometry between the Cayley graphs $\left(G, S_{1}\right)$ and $\left(G, S_{2}\right)$. At the beginning of the 80's, M. Gromov (see [6]) initiated the study of finitely generated groups up to quasi-isometry.

Example 1.7. The universal cover of a compact Riemannian manifold is quasi-isometric to every Cayley graph of the covering group (see [6] and $[\mathbf{1 2}])$.

Note that the notion of quasi-isometry is purely metric. So, when we look for quasi-isometry invariant properties of metric measure spaces, we are led to assume some uniformity properties on the volume of balls. This is the reason why, for instance, this notion is well adapted to geometric group theory. But since we want to deal with more general spaces, we will define a more restrictive class of maps. Those maps will be asked to preserve locally the volume of balls. On the other hand, we want local properties to be stable under bilipschitz fluctuations of the metric. Precisely, let $(X, d, \mu)$ be a metric measure space and let $d^{\prime}$ be another metric on $X$ such that $d / d^{\prime}$ and $d^{\prime} / d$ are bounded. The following definition (see [1]) prevents wild changes of the volume of balls with bounded radii under the identity map between $(X, d, \mu)$ and $\left(X, d^{\prime}, \mu\right)$.

Definition 1.8. Let us say that $(X, d, \mu)$ is doubling at fixed radius, or has property $(D V)_{\text {loc }}$ if for all $r>0$, there exists $C_{r}>0$ such that, for all $x \in X$

$$
\mu(B(x, 2 r)) \leq C_{r} \mu(B(x, r)) .
$$

Remark 1.9. Note that property $(D V)_{\text {loc }}$ is local in $r$ but uniform in $x$.

Example 1.10. Bounded degree graphs or Riemanniann manifolds with Ricci curvature bounded from below satisfy $(D V)_{\text {loc }}$.

The following notion was introduced by Kanai [8] (see also [1]).

Definition 1.11. Let $(X, d, \mu)$ and $\left(X^{\prime}, d^{\prime}, \mu^{\prime}\right)$ be two metric measure spaces with property $(D V)_{\text {loc }}$. Let us say that $X$ and $X^{\prime}$ are large scale equivalent (we can easily check that it is an equivalence relation) if there is a function $f$ from $X$ to $X^{\prime}$ with the following properties: there exist some constants $C_{1}>0, C_{2} \geq 1, C_{3} \geq 1$ such that 
(a) $f$ is a quasi-isometry of constants $C_{1}$ and $C_{2}$;

(b) for all $x \in X$

$$
C_{3}^{-1} \mu(B(x, 1)) \leq \mu^{\prime}(B(f(x), 1)) \leq C_{3} \mu(B(x, 1)) .
$$

Focusing our attention on balls of radius 1 may not seem very natural. Nevertheless, this is not a serious issue since property $(D V)_{\text {loc }}$ allows to make no distinction between balls of radius 1 and balls of radius $C$ for any constant $C>0$.

Remark 1.12. Note that for graphs with bounded degree (equipped with the counting measure), or Riemannian manifolds with bounded Ricci curvature (equipped with the Riemannian measure), quasi-isometries are automatically large-scale equivalences.

\subsection{Volume of balls and growth function.}

Let $(X, d, \mu)$ be a metric measure space. The equivalence class modulo $\approx$ of $\mu(B(x, r))$ does not depend on $x$. We call it the volume growth of $X$ and we write it $V(r)$. We have the following easy fact (see [1] ).

Proposition 1.13. The volume growth is invariant under large-scale equivalence (among $(D V)_{\text {loc }}$ spaces).

Definition 1.14. Let $X$ be a metric measure space. We say that $X$ is doubling if there exists a constant $C>0$ such that, $\forall x \in X$ and $\forall r \geq 0$

$$
\mu(B(x, 2 r)) \leq C \mu(B(x, r)) .
$$

We will call this property $(D V)$.

Remark 1.15. It is easy to see that $(D V)$ is invariant under large scale equivalence between $(D V)_{\text {loc }}$ spaces. To be more general, we could define an asymptotic doubling condition $(D V)_{\infty}$, restricting (1.1) to balls of radius more than a constant (depending on the space). Property $(D V)_{\infty}$ is also stable under large-scale equivalence between $(D V)_{\text {loc }}$ spaces and has the advantage to focus on large scale properties only. Actually, in every situation met in this paper, the assumption $(D V)$ can be replaced by $(D V)_{\infty}+(D V)_{\text {loc }}$ (note that they are equivalent for graphs). Nevertheless, for the sake of simplicity, we will leave this generalization aside.

Example 1.16. A crucial class of doubling spaces is the class of spaces with polynomial growth: we say that a metric measure space has (strict) polynomial growth of degree $d$ if there exists a constant $C \geq 1$ such that, $\forall x \in X$ and $\forall r \geq 1$

$$
C^{-1} r^{d} \leq \mu(B(x, r)) \leq C r^{d} .
$$


Gromov proved [5] that if a finitely generated group $G$ satisfies

$$
\mu(B(1, r)) \leq C r^{d}
$$

for some constant $C>0$, then it has polynomial growth with integer degree. Another very interesting class of examples are fractals as for instance, the (unbounded) Sierpinski gasket or more generally, polygaskets (see $[\mathbf{4}],[\mathbf{1 3}])$.

\section{Organization of the paper}

In the next section, we present a setting adapted to the study of asymptotic isoperimetry in general metric measure spaces. The main interest of this setting is that the "asymptotic isoperimetric properties" are invariant under large-scale equivalence. In particular, it will imply that if $X$ is a $(D V)_{\text {loc }}$ and uniformly connected space (see next section), then the class modulo $\approx$ of $I_{h}$ will not depend any more on $h$ provided $h$ is large enough. For that reason, we will simply denote $I$ instead of $I_{h}$. Then, we introduce a notion of weak geodesicity which is invariant under Hausdorff equivalence (see Section 3.2) but not under quasi-isometry. We call it property $(\mathrm{M})$ since it can be formulated in terms of existence of some "monotone" geodesic chains between any pair of points. This property plays a crucial role when we want to obtain upper bounds for the volume of spheres (see [14]). It will also appear as a natural condition for some properties discussed in this paper.

Here are the two main problems concerning isoperimetry in metric measure spaces: first, determining the asymptotic behavior of the profile; second, finding families of subsets that optimize the profile. The asymptotic behavior of $I$ is more or less related to volume growth (see [2] and [9] for the case of finitely generated groups). In the setting of groups, the two problems have been solved for Lie groups (and for polycyclic groups) in [10] and [2] and for a wide class of groups constructed by wreath products in [3]. It seems very difficult (and probably desperate) to get general statements for graphs with bounded degree without any regularity assumption (like doubling property or homogeneity). On the other hand, let us emphasize the fact that doubling condition appears as a crucial assumption in many fields of analysis. So in this article, we will deal essentially with doubling metric measure spaces. Without any specific assumption on the space, balls seem to be natural candidates for being isoperimetric subsets, especially when the space is doubling (see Corollary 4.4).

One could naively think that thanks to Theorem 3.10, a property like asymptotic isoperimetry of balls is stable under large-scale equivalence. 
Unfortunately, it is not the case: this is essentially due to the fact that the image of a ball under a quasi-isometry is quite far from being a ball. Namely, in order to apply Theorem 3.10, one would need the existence of some $C>0$ such that

$$
\begin{aligned}
& B(f(x), r-C) \subset[f(B(x, r))]_{C} \subset B(f(x), r+C) \\
& \forall x \in X, \forall r>0 .
\end{aligned}
$$

This condition is satisfied if $f$ is a Hausdorff ${ }^{1}$ equivalence. But if $f$ is only a quasi-isometry, one cannot expect better than the following inclusions

$$
\begin{array}{r}
B\left(f(x), C^{-1} r-C\right) \subset[f(B(x, r))]_{C} \subset B(f(x), C r+C) \\
\forall x \in X, \forall r>0 .
\end{array}
$$

Let us introduce some terminology. First, let us write $\mathcal{B}$ for the family of all closed balls of $X$.

Definition 2.1. Let $X$ be a metric measure space.

- We say that $X$ is (IB) if balls are asymptotically isoperimetric, i.e. if

$$
I_{\mathcal{B}}^{\downarrow} \preceq I .
$$

Otherwise, we will say that $X$ is (NIB).

- We say that $X$ is strongly-(IB) if balls are strongly asymptotically isoperimetric, i.e. if

$$
I_{\mathcal{B}}^{\uparrow} \preceq I .
$$

- Finally, we say that a metric measure space is stably-(IB) (resp. stably-(NIB)) if every space with ${ }^{2}$ Property (M) that is large scale equivalent to $X$ is (IB) (resp. (NIB)).

Definition 2.2. We say that a space $(X, d, \mu)$ satisfies a strong (isoperimetric) inequality —or that $X$ has a strong profile - if $I \succeq$ id $/ \phi$ where $\phi$ is the equivalence class modulo $\approx$ of the function

$$
t \rightarrow \inf \{r, \mu(B(x, r) \geq t\} .
$$

\footnotetext{
${ }^{1}$ See Section 3.2 for a definition.

${ }^{2}$ Property $(\mathrm{M})$ is an abreviation for "monotone geodesic property" which is slightly weaker than being geodesic, see Definition 3.15.
} 
We will show that every doubling space satisfying a strong isoperimetric inequality satisfies (IB). This actually implies that such a space satisfies stably-(IB). In particular, any compactly generated, locally compact group of polynomial growth satisfies (IB). In contrast, apart from the Abelian case [14], it is still unknown whether such a group $G$ satisfies strongly-(IB) or not, or, in other words, if we have $\mu\left(K^{n+1} \backslash K^{n}\right) \approx n^{d-1}$ where $K$ is a compact generating set of $G$ and $\mu$ is a Haar measure on $G$.

Conversely, we will show that every strongly-(IB) doubling space satisfies a strong isoperimetric inequality. On the other hand, we will see that the strong isoperimetric inequality does not imply strongly-(IB), even if the volume growth is linear $(V(r) \approx r)$.

To see that strongly-(IB) is not stable under large scale equivalence, even among graphs with polynomial growth, we shall construct a graph quasi-isometric to $\mathbf{Z}^{2}$ whose volume of spheres is not dominated by $r^{\log 3 / \log 2}$ (where $r$ is the radius). Note that this can be compared with the following result (see [14, Theorem 1]).

Theorem $2.3([\mathbf{1 4}])$. Let $X$ be a metric measure space with properties $(M)$ and $(D V)$ (for instance, a graph or a complete Riemannian manifold with the doubling property). There exists $\delta>0$ and a constant $C>0$ such that, $\forall x \in X$ and $\forall r>0$

$$
\mu(B(x, r+1) \backslash B(x, r)) \leq C r^{-\delta} \mu(B(x, r)) .
$$

In particular, the ratio $\mu\left(\partial B_{x, r}(x)\right) / \mu(B(x, r))$ tends to 0 uniformly in $x$ when $r$ goes to infinity.

When the profile is not strong, we will see that many situations can happen. All the counterexamples built in the corresponding section will be graphs of polynomial growth.

The case of a bounded profile is quite specific. ${ }^{3}$ Indeed, in that case, and under some hypothesis on $X$ (including graphs and manifolds with bounded geometry), we will prove that if $\left(P_{n}\right)_{n \in \mathbf{N}}$ is an asymptotically isoperimetric sequence of connected subsets of $X$, one can find a constant $C \geq 1$ and $\forall n \in \mathbf{N}$, some $x_{n} \in X, r_{n}>0$ such that

$$
B\left(x_{n}, r_{n}\right) \subset P_{n} \subset B\left(x_{n}, C r_{n}\right) .
$$

Note that here, we don't ask $X$ to be doubling.

\footnotetext{
${ }^{3}$ Note that there exist infinite self-similar graphs such as the unbounded Serpinsky gasket [13], with polynomial growth and with bounded asymptotic isoperimetric profile.
} 
Nevertheless, we will see that there exist graphs with polynomial growth (with unbounded profile) such that no asymptotically isoperimetric family has this property. In particular, those graphs are stably-(NIB).

To be complete, we also build graphs with polynomial growth, bounded profile and satisfying stably-(NIB).

Concerning the stability under large-scale equivalence, we will see that even among graphs with polynomial growth, with bounded or unbounded profile, property (IB) is not stable under large-scale equivalence (in the case of graphs equipped with the counting measure, a large-scale equivalence is simply a quasi-isometry).

Finally, we shall examine isoperimetric properties of connected subsets.

Definition 2.4. Let us say that a subset $A$ is (metrically) connected if for any partition $A=A_{1} \sqcup A_{2}$ such that $\partial A_{1} \cap \partial A_{2}=\emptyset$, either $A_{1}$ or $A_{2}$ is empty.

Clearly, since balls of a (M)-space are connected, the strong isoperimetric inequality implies that connected sets are asymptotically isoperimetric (see also Theorem 6.1).

On the other hand, we will show that there exist graphs with polynomial growth whose connected subsets are not asymptotically isoperimetric: namely there exists an increasing sequence of integers $\left(N_{n}\right)$ such that to optimize (asymptotically) the isoperimetric profile at these values, one has to take a sequence of subsets with an number of connected components that tends to infinity and such that the distance between these connected components also tends to infinity.

Remark 2.5. Note that all our conterexamples are far from being homogeneous. So many of the properties discussed in this paper should also be discussed in a more restrictive class of spaces such as spaces with fractal properties.

\section{Isoperimetry at infinity: a general setting}

\subsection{Isoperimetric at a given scale.}

The purpose of this section is to find some minimal conditions under which "isoperimetric properties at infinity" are invariant under largescale equivalence. In the introduction, namely in Section 1.1, we justified our definition of the boundary by the fact that we want it to have a uniform thickness. Nevertheless, it is not suffisant to our purpose: we will also need a discrete connectivity property. Indeed, let $X$ be a graph; 
if $h=1 / 2$, then every subset of $X$ has a trivial boundary, so that all the isoperimetric properties of $X$ are trivial.

Definition 3.1. Let $X$ be a metric space and fix $b>0$. Let us call a $b$-chain of length $n$ from $x$ to $y$, a finite sequence $x_{0}=x, \ldots, x_{n}=y$ such that $d\left(x_{i}, x_{i+1}\right) \leq b$.

The following definition can be used to study the isoperimetry at a given scale, although we will only use it "large-scale version" in this paper.

\section{Definition 3.2.}

Scaled version: Let $b>0$ and $E_{1} \gg b$. Let us say that $X$ is uniformly $b$-connected at scale $\leq E_{1}$ if there exists a constant $E_{2} \geq E_{1}$ such that for every couple $x, y \in X$ such that $d(x, y) \leq E_{1}$, there exists a $b$-chain from $x$ to $y$ totally included in $B\left(x, E_{2}\right)$.

Large-scale version: If, for all $E_{1} \gg b, X$ is uniformly $b$-connected at scale $\leq E_{1}$, then we say that $X$ is uniformly $b$-connected (or merely uniformly connected).

Remark 3.3. Note that in the scaled version, the space $X$ is allowed to have a proper nonempty subset $A$ such that $d\left(A, A^{c}\right)>E_{1}$ : in this case $X$ is not $b$-connected at all.

Invariance under quasi-isometry. Note that if $X$ is uniformly $b$-connected at scale $\leq E_{1}$ and if $f: X \rightarrow X^{\prime}$ is a quasi-isometry of constants $C_{1}$ and $C_{2}$, then $X^{\prime}$ is uniformly $C_{2} b+C_{1}$-connected at scale $\leq$ $E_{1} / C_{2}-C_{1}$. In particular, if $X$ is uniformly $b$-connected, then $X^{\prime}$ is uniformly $\left(C_{2} b+C_{1}\right)$-connected.

Remark 3.4. Let us write $d_{b}(x, y)$ for the $b$-distance from $x$ to $y$, that is, the minimal length of a $b$-chain between $x$ and $y$ (note that if every couple of points of $X$ can be joined by a $b$-chain, then $d_{b}$ is a pseudometric on $X$ ).

If there ${ }^{4}$ exists $C>0$ such that, for all $x, y \in X$, one has $d_{b}(x, y) \leq$ $C d(x, y)+C$, then in particular, $X$ is uniformly $b$-connected.

Example 3.5. A graph and a Riemannian manifold are respectively uniformly 1-connected and uniformly $b$-connected for all $b>0$.

\footnotetext{
${ }^{4}$ Such a space is often called $b$-quasi-geodesic.
} 
Proposition 3.6. Let $X$ be a uniformly b-connected space at scale $\leq E_{1}$. Let $h$ be such that $h \geq 2 b$.

(i) For every subset $A$ of $X$ and every $x \in A^{c}$ such that $d(x, A)<E_{1}$ (resp. $x \in A$ such that $\left.d\left(x, A^{c}\right)<E_{1}\right)$, there exists a point $z \in \partial_{h} A$ at distance $\leq E_{2}$ of $x$ such that

$$
B(z, b) \subset \partial_{h} A .
$$

(ii) If, moreover, $X$ is $(D V)_{\text {loc }}$ and $h \ll E_{1}$, then there exists a constant $C^{\prime} \geq 1$ such that, for every subset $A$, there exists a family $\left(B\left(y_{i}, b\right)\right)_{i}$ included in $\partial_{h} A$, such that, for all $i \neq j, d\left(y_{i}, y_{j}\right) \geq$ $E_{2}$ and such that

$$
\sum_{i} \mu\left(B\left(y_{i}, b\right)\right) \leq \mu\left(\partial_{h} A\right) \leq C^{\prime} \sum_{i} \mu\left(B\left(y_{i}, b\right)\right) .
$$

(iii) The h-boundary measure of a subset of a $(D V)_{\mathrm{loc}}$, uniformly b-connected space does not depend on $h$ up to a multiplicative constant, provided $E_{1} \gg h \geq 2 b$.

Proof: Let $x \in A^{c}$ such that $d(x, A)<E_{1}$ and let $y \in A$ be such that $d(x, y) \leq E_{1}$. We know from the hypothesis that there exists a finite chain $x_{0}=x, x_{1}, \ldots, x_{n}=y$ satisfying

- $x_{n} \in A$,

- $d\left(x, x_{i}\right) \leq E_{2}$ for all $i$,

- for all $1 \leq i \leq n, d\left(x_{i-1}, x_{i}\right) \leq b$.

Since $x \in A^{c}$ and $y \in A$, there exists $j \leq n$ such that $x_{j-1} \in A^{c}$ and $x_{j} \in A$. Clearly, $x_{j} \in A_{b} \cap\left[A^{c}\right]_{b}=\partial_{b} A$. But since $\left[\partial_{b} A\right]_{b} \subset \partial_{2 b} A \subset \partial_{h} A$, the ball $B\left(x_{j}, b\right)$ is included in $\partial_{h} A$, which proves the first assertion.

Let us show the second assertion. Consider a maximal family of disjoint balls $\left(B\left(x_{i}, 2 E_{2}\right)\right)_{i \in I}$ with centers $x_{i} \in \partial_{h} A$. Then $\left(B\left(x_{i}, 5 E_{2}\right)\right)_{i \in I}$ forms a covering of $\partial_{h} A$.

Using the first assertion and the fact that $h \ll E_{1}$, one sees that each $B\left(x_{i}, 2 E_{2}\right)$ contains a ball $B\left(y_{i}, b\right)$ included in $\partial_{h} A$. It is clear that the balls $B\left(y_{i}, 10 E_{2}\right)$ form a covering of $\partial_{h} A$ and that the balls $\left(B\left(y_{i}, b\right)\right.$ are disjoint. But, by property $(D V)_{\text {loc }}$, there exists $C^{\prime} \geq 1$, depending on $b$ and $E_{2}$, such that, for all $i \in I$

$$
\mu\left(B\left(y_{i}, 10 E_{2}\right)\right) \leq C^{\prime} \mu\left(B\left(y_{i}, b\right)\right) .
$$

We deduce

$$
\sum_{i} \mu\left(B\left(y_{i}, b\right)\right) \leq \mu\left(\partial_{h} A\right) \leq C^{\prime} \sum_{i} \mu\left(B\left(y_{i}, b\right)\right)
$$

which proves (ii). The assertion (iii) now follows from (ii). 
Remark 3.7. This proposition gives conditions to study isoperimetry at scale between $b$ and $E_{1}$, i.e. choosing $h$ far from those two bounds. Thus, we will always assume that this condition holds and we will simply write $\partial A$ instead of $\partial_{h} A$. Otherwise, problems may happen. We talked about what can occur if $h<b$ at the beginning of this section. Now, let us give an idea of what can happen if $h>E_{1}$. Consider a metric measure space $X$ such that $X=\cup_{i \in I} X_{i}$ where the $X_{i}$ are subsets such that $d\left(X_{i}, X_{j}\right) \geq E_{1}$ whenever $i \neq j$ and such that $\mu\left(X_{i}\right)$ is finite for every $i \in I$ but not bounded. Note that for $h<E_{1}$ the boundary of every $X_{i}$ is empty so that the family $\left(X_{i}\right)_{i \in I}$ is trivially asymptotically isoperimetric. But this can change dramatically if $h>E_{1}$ because the boundary of $X_{i}$ can meet many $X_{j}$ 's for $j \neq i$.

Remark 3.8. If we replace uniformly $b$-connected at scale $\leq E_{1}$ by uniformly $b$-connected, then the proposition gives a setting adapted to the study of large scale isoperimetry. Namely, it says that for a uniformly $b$-connected, $(D V)_{\text {loc }}$ space, the choice of $h$ does not matter, provided $h \geq 2 b$.

Corollary 3.9. Let $X$ be a $(D V)_{\mathrm{loc}}$, uniformly b-connected space. If $h, h^{\prime} \geq 2 b$, we have

$$
I_{h} \approx I_{h^{\prime}}
$$

So, from now on, we will simply call "profile" (instead of $h$-profile) the equivalence class modulo $\approx$ of $I_{h}$. Note that the same holds for restricted profiles $I_{h, \mathcal{A}}^{\downarrow}$, and $I_{h, \mathcal{A}}^{\uparrow}$ that we will simply denote $I_{\mathcal{A}}^{\downarrow}$ and $I_{\mathcal{A}}^{\uparrow}$ (where $\mathcal{A}$ is a family of subsets of $X$ ).

The following theorem shows that a large-scale equivalence $f$ with controlled constants essentially preserves all isoperimetric properties.

Theorem 3.10. Let $f(X, d, \mu) \rightarrow\left(X^{\prime}, d^{\prime}, \mu^{\prime}\right)$ be a large-scale equivalence (with constants $C_{1}, C_{2}$ and $C_{3}$ ) where $X$ (resp. $\left.X^{\prime}\right)$ is $(D V)_{\text {loc }}$ and uniformly $b$-connected at scale $\leq E_{1}$ (resp. uniformly $b^{\prime}$-connected at scale $\left.\leq E_{1}^{\prime}\right)$. We suppose also that $E_{1}$ and $E_{1}^{\prime}$ are far larger than $C_{1}$, $C_{2}, C_{2} b$ and $C_{2}\left(b^{\prime}+C_{1}\right)$. Then, there exists a constant $K \geq 1$ such that, for any subset $A$ of finite measure

$$
\mu^{\prime}\left(\partial[f(A)]_{C_{1}}\right) \leq K \mu(\partial A) .
$$

Proof: Let us start with a lemma.

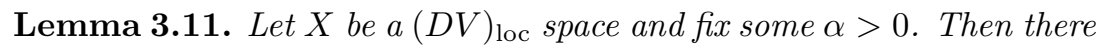
exists a constant $c>0$ such that, for all family $\left(B\left(x_{i}, \alpha\right)\right)_{i \in I}$ of disjoint balls of $X$, there is a subset $J$ of $I$ such that $\forall j \in J$, the balls $B\left(x_{j}, 2 \alpha\right)$ 
are still disjoint, and such that

$$
\sum_{j \in J} \mu\left(B\left(x_{j}, 2 \alpha\right)\right) \geq c \sum_{i \in I} \mu\left(B\left(x_{i}, \alpha\right)\right) .
$$

Proof: Let us consider a maximal subset $J$ of $I$ such that $\left(B\left(x_{j}, 2 \alpha\right)\right)_{j \in J}$ forms a family of disjoint balls. Then, by maximality, we get

$$
\bigcup_{i \in I} B\left(x_{i}, \alpha\right) \subset \bigcup_{j \in J} B\left(x_{j}, 4 \alpha\right) .
$$

We conclude thanks to property $(D V)_{\mathrm{loc}}$.

To fix ideas, take $h=2 b$ and $h^{\prime}=2 b^{\prime}$. Assertion (ii) of Proposition 3.6 implies that there exists a family of balls $\left(B\left(y_{i}, b^{\prime}\right)\right)_{i}$ included in $\partial[f(A)]_{C_{1}}$ such that, for all $i \neq j, d\left(y_{i}, y_{j}\right) \geq E_{2}^{\prime}$ and such that

$$
\sum_{i} \mu\left(B\left(y_{i}, b^{\prime}\right)\right) \leq \mu\left(\partial_{h}[f(A)]_{C_{1}}\right) \leq C^{\prime} \sum_{i} \mu\left(B\left(y_{i}, b^{\prime}\right)\right) .
$$

By the lemma, and up to changing the constant $C^{\prime}$, one can even suppose that $d\left(y_{i}, y_{j}\right) \gg C_{2} E_{2}$ for $i \neq j$.

For all $i$, let $x_{i}$ be a element of $X$ such that $d\left(f\left(x_{i}\right), y_{i}\right) \leq C_{1}$. The points $x_{i}$ are then at distance $\gg E_{2}$ to one another. Moreover, since $y_{i}$ is both at distance $\leq 2 b+C_{1}$ of $f(A)$ and of $f\left(A^{c}\right), x_{i}$ is both at distance $\ll E_{1}$ of $A$ and of $A^{c}$. So, by the assertion (i) of the proposition, there exists a ball $B\left(z_{i}, b\right)$ included in $\partial A \cap B\left(x_{i}, E_{2}\right)$. Since balls $B\left(x_{i}, E_{2}\right)$ are disjoint, so are the $B\left(z_{i}, b\right)$. The theorem then follows from property $(D V)_{\text {loc }}$ and from property of "almost-conservation" of the volume (property (b)) of large-scale equivalence.

Remark 3.12. Note that in the case of graphs, the condition $h \geq 2$ can be relaxed to $h \geq 1$ (the proposition and the theorem stay true and their proofs are unchanged).

Corollary 3.13. Under the hypotheses of the theorem, we have

(i) if the family $\left(A_{i}\right)_{i \in I}$ is asymptotically isoperimetric, then so is $\left(f\left(A_{i}\right)_{b}\right)_{i \in I}$

(ii) if $I$ and $I^{\prime}$ are the profiles of $X$ and $X^{\prime}$ respectively, we get $I \approx I^{\prime}$.

The corollary results immediately from the theorem and the following proposition. 
Proposition 3.14. Let $f$ be a large-scale equivalence between two $(D V)_{\text {loc }}$ spaces $X$ and $X^{\prime}$. Then for every subset $A$ of $X$, there exists $C \geq 1$ such that

$$
\mu(A) \leq C \mu^{\prime}\left([f(A)]_{C_{1}}\right) .
$$

Proof: Consider a maximal family of disjoint balls $\left(B\left(y_{i}, C_{1}\right)\right)_{i \in I}$ whose centers belong to $f(A)$. These balls are clearly included in $[f(A)]_{C_{1}}$. By property $(D V)_{\text {loc }}$, the total volume of these balls, and therefore $\mu^{\prime}\left([f(A)]_{C_{1}}\right)$, are comparable to the sum of the volumes of balls $B\left(x_{i}, 3 C_{1}\right)_{i \in I}$ that form a covering of $[f(A)]_{C_{1}}$. The preimages of these balls thus cover $A$. But, for each $i, f^{-1}\left(B\left(y_{i}, 3 C_{1}\right)\right)$ is contained in a ball of radius $3 C_{1} C_{2}+C_{2}$ and of center $x_{i}$ where $x_{i} \in f^{-1}\left(\left\{y_{i}\right\}\right)$. By property $(D V)_{\text {loc }}$ and property of almost-conservation of the measure of small balls (property (b)) of $f$, the measure of this ball is comparable to that of $B\left(y_{i}, 3 C_{1}\right)$. So we are done.

Finally, let us mention that if we suppose that $X$ and $X^{\prime}$ are uniformly connected and satisfy the $(D V)_{\text {loc }}$ condition, then Theorem 3.10 and its corollary hold for any large-scale equivalence $f$.

\subsection{Property (M): monotone geodesicity.}

Let us introduce a natural (but quite strong) property of geodesicity.

Definition 3.15. Let us say that $(X, d)$ has property (M) if there exists $C \geq 1$ such that, $\forall x \in X, \forall r>0$ and $\forall y \in B(x, r+1)$, we have $d(y, B(x, r)) \leq C$.

Remark 3.16. Let $(X, d)$ be a $(\mathrm{M})$ metric space. Then $X$ has "monotone geodesics" (this is why we call this property (M)): i.e. there exists $C \geq 1$ such that, for all $x, y \in X$, there exists a finite chain $x_{0}=x, x_{1}, \ldots, x_{n}=y$ such that $\forall 0 \leq i<n$,

$$
d\left(x_{i}, x_{i+1}\right) \leq C ;
$$

and

$$
d\left(x_{i}, x\right) \leq d\left(x_{i+1}, x\right)-1 .
$$

Consequently, $\forall r, k>0, \forall y \in B(x, r+k)$, we have

$$
d(y, B(x, r)) \leq C k .
$$

These two properties are actually trivially equivalent to property (M).

Recall (see [7, p. 2]) that two metric spaces $X$ and $Y$ are said Hausdorff equivalent

$$
X \sim_{\text {Hau }} Y
$$


if there exists a (larger) metric space $Z$ such that $X$ and $Y$ are contained in $Z$ and such that

$$
\sup _{x \in X} d(x, Y)<\infty
$$

and

$$
\sup _{y \in Y} d(y, X)<\infty .
$$

Remark 3.17. It is easy to see that property $(\mathrm{M})$ is invariant under Hausdorff equivalence. But on the other hand, property (M) is unstable under quasi-isometry. To construct a counterexample, one can quasiisometrically embed $\mathbf{R}_{+}$into $\mathbf{R}^{2}$ such that the image, equipped with the induced metric does not have property $(\mathrm{M})$ : consider a curve starting from 0 and containing for every $k \in \mathbf{N}$ a half-circle of radius $2^{k}$. So it is strictly stronger than quasi-geodesic property $[\mathbf{7}$, p. 7$]$, which is invariant under quasi-isometry: $X$ is quasi-geodesic if there exist two constants $d>0$ and $\lambda>0$ such that for all $(x, y) \in X^{2}$ there exists a finite chain of points of $X$

$$
x=x_{0}, \ldots, x_{n}=y,
$$

such that

$$
d\left(x_{i-1}, x_{i}\right) \leq d, \quad i=1, \ldots, n,
$$

and

$$
\sum_{i=1}^{n} d\left(x_{i-1}, x_{i}\right) \leq \lambda d(x, y) .
$$

Example 3.18. A geodesic space has property $(\mathrm{M})$, in particular graphs and complete Riemannian manifolds have property (M). A discretisation (i.e. a discrete net) of a Riemannian manifold $X$ has property (M) for the induced distance.

Remark 3.19. Note that in general, if $X$ is a metric measure space, we have

$$
\partial_{1 / 2} B(x, r+1 / 2) \subset B(r+1) \backslash B(x, r) .
$$

Moreover, if $X$ has property $(\mathrm{M})$, then, we have

$$
B(x, r+1) \backslash B(x, r) \subset \partial_{C} B(x, r+1) .
$$

Note that this is not true in general, even for quasi-geodesic spaces. 


\section{Link between isoperimetry of balls and strong isoperimetric inequality}

\subsection{Strong isoperimetric inequality implies (IB).}

The spaces we will consider from now on will be $(D V)_{\text {loc }}$ and uniformly 1-connected. Let us write $\partial A=\partial_{2} A$ for any subset $A$ of a metric space $X$ (note that these conventions are motivated by Proposition 3.6).

Let $X$ be a metric measure space. Let $V$ be a nondecreasing function belonging to the volume growth class (for instance $V(r)=\mu(B(x, r)$ ) for a $x \in X)$. Write $\phi(t)=\inf \{r, V(r) \geq t\}$ for the "right inverse" function of $V$. Remark that if $f$ and $g$ are nondecreasing functions $\mathbf{R}_{+} \rightarrow \mathbf{R}_{+}$, then $f \approx g$ if and only if their right inverses are equivalent. In particular, the equivalence class of $\phi$ is invariant under large-scale equivalence.

Definition 4.1. Let us call a strong isoperimetric inequality the following kind of isoperimetric inequality

$$
\forall A \subset X, \quad|\partial A| \geq C^{-1}|A| / \phi(C|A|) .
$$

Remark that this is equivalent to

$$
I \succeq \operatorname{id} / \phi .
$$

Therefore, if $X$ satisfies a strong isoperimetric inequality, we will say that it has a strong profile.

Example 4.2. If $X$ has polynomial growth of degree $d$, we have $\phi(t) \approx$ $t^{1 / d}$. So $X$ has a strong profile if and only if

$$
I \succeq(\mathrm{id})^{\frac{d-1}{d}} .
$$

Write, for all $x \in X$ and for all $0<r<r^{\prime}$

$$
C_{r, r^{\prime}}(x)=B\left(x, r^{\prime}\right) \backslash B(x, r) .
$$

Proposition 4.3. Let $X$ be a doubling space (here, no other hypothesis is required). There exists a constant $C \geq 1$ such that

$$
\forall x \in X, \forall r \geq 1, \quad \inf _{r \leq r^{\prime} \leq 2 r} \mu\left(C_{r^{\prime}-1, r^{\prime}}\right) \leq C \mu(B(x, r)) / r .
$$

Proof: Clearly, it suffices to prove the proposition when $r=n$ is a positive integer. First, note that

$$
\cup_{k=n}^{2 n}(B(x, k) \backslash B(x, k-1)) \subset B(x, 2 n) .
$$

So, we have

$$
\mu(B(x, 2 n)) \geq n \inf _{n \leq k \leq 2 n} \mu(B(x, k) \backslash B(x, k-1)) .
$$

We conclude by doubling property. 
Corollary 4.4. Let $X$ be a uniformly connected doubling space. Then we have

$$
I_{\mathcal{B}}^{\downarrow} \preceq \mathrm{id} / \phi .
$$

Namely, there exists a constant $C \geq 1$ such that

$$
\forall x \in X, \forall r>0, \quad \inf _{r^{\prime} \geq r} \mu\left(\partial B\left(x, r^{\prime}\right)\right) \leq C \mu(B(x, r)) / r .
$$

Proof: This follows from Remark 3.19.

Corollary 4.5. Let $X$ be a uniformly connected doubling space satisfying a strong isoperimetric inequality. Then, $X$ is stably-(IB).

Proof: It follows from Corollary 4.4 and from Corollary 3.13.

Remark 4.6. Varopoulos [15] showed that the strong isoperimetric inequality is satisfied by any group of polynomial growth. Coulhon and Saloff-Coste [2] then proved it for any unimodular compactly generated locally compact group with a simple and elegant demonstration. We have the following corollary.

Corollary 4.7. A Cayley graph of a group of polynomial growth is stably-(IB).

\subsection{The strong isoperimetric inequality does not imply strong- ly-(IB).}

Note that this will result from the example shown in Section 4.3. Let us present here a counterexample with linear growth.

For every integer $n$, we consider the following finite rooted tree $G_{n}$ : first take the standard binary tree of depth $n$. Then stretch it as follows: replace each edge connecting a $k-1$ 'th generation vertex to a $k$ 'th generation vertex by a (graph) interval of length $2^{2^{n-k}}$. Then consider the graph $G_{n}^{\prime}$ obtained by taking two copies of $G_{n}$ and identifying the vertices of last generation of the first copy with those of the second copy. Write $r_{n}$ and $r_{n}^{\prime}$ for the two vertices of $G_{n}^{\prime}$ corresponding to the respective roots of the two copies of $G_{n}$. Finally, glue "linearly" the $G_{n}^{\prime}$ together identifying $r_{n}^{\prime}$ with $r_{n+1}$, for all $n$ : it defines a graph $X$.

Let us show that $X$ has linear growth (i.e. polynomial growth of degree 1 ). Thus $I \approx 1$, and since the boundary volume of balls is clearly not bounded, we do not have $I_{B}^{\uparrow} \preceq I$. In particular, $X$ is not strongly-(IB).

Since $X$ is infinite, it is enough to show that there exists a constant $C>0$ such that

$$
|B(x, r)| \leq C r
$$


for every vertex $x$ of $X$. But it is clear that among the balls of radius $r$, those which are centered in points of $n$ 'th generation of a $G_{n}$ for $n$ large enough are of maximal volume. Let us take such an $x$. Remark that for $\sum_{j=0}^{k} 2^{2^{j}} \leq r \leq \sum_{j=0}^{k+1} 2^{2^{j}}$, we have

$$
|B(x, r)| \leq 2\left|B\left(x, \sum_{j=0}^{k} 2^{2^{j}}\right)\right|+2\left(r-\sum_{j=0}^{k} 2^{2^{j}}\right) .
$$

So it is enough to show (4.1) for $r=\sum_{j=0}^{k} 2^{2^{j}}$. We have

$$
\mu\left(B\left(x, \sum_{j=0}^{k} 2^{2^{j}}\right)\right)=\sum_{j=0}^{k} 2.2^{j} \cdot 2^{2^{k-j}} \leq 4.2^{2^{k}} .
$$

Which proves (4.1) with $C=8$.

Remark 4.8. This example and that of Section 4.3 show in particular that the strong isoperimetric inequality does not imply (even in linear growth case) strongly-(IB).

\subsection{Instability of strongly-(IB) under quasi-isometry.}

Theorem 4.9. We can find a graph, quasi-isometric to $\mathbf{Z}^{2}$ (resp. a Riemannian manifold $M$ bi-Lipschitz equivalent to $\mathbf{R}^{2}$ ) whose volume of spheres is not dominated by $r^{\log 3 / \log 2}$ (where $r$ is the radius).

Remark 4.10. The restriction to dimension 2 is not essential, but was made to simplify the exposition (actually, we merely need the dimension to be greater or equal to 2 ).

Proof: The general idea of the construction is to get a sequence of spheres which look like finitely iterated Von Koch curves. First, we will build a graph with weighted edges. Actually, this graph will be simply the standard Cayley graph of $\mathbf{Z}^{2}$, and the edges will have lengths equal to 1 except for some selected edges which will have length equal to a small, but fixed positive number.

First step of the construction: Let us define a sequence $\left(A_{k}\right)$ of disjoint subtrees of $\mathbf{Z}^{2}$ (which is identified to its usual Cayley graph). Let $\left(e_{1}, e_{2}\right)$ be the canonical basis of $\mathbf{Z}^{2}$ and denote $S=\left\{ \pm e_{1}, \pm e_{2}\right\}$. For every $k \geq 1$, let $a_{k}=\left(2^{2 k}, 0\right)$ be the root of the tree $A_{k}$ and define $A_{k}$ by

$$
\begin{aligned}
& x \in A_{k} \Leftrightarrow x=a_{k}+2^{k} \varepsilon_{0}(x)+2^{k-1} \varepsilon_{1}(x)+\cdots \\
& \cdots+2^{k-i(x)} \varepsilon_{i(x)}(x)+r(x) \varepsilon_{i(x)+1}(x)
\end{aligned}
$$


where

$-0 \leq i(x) \leq k-1$,

$-\varepsilon_{j}(x)$ belongs to $S$ for every $0 \leq j \leq i(x)+1$ and is such that $\varepsilon_{j+1}(x) \neq-\varepsilon_{j}(x)($ for $j \leq i(x))$,

$-r(x) \leq 2^{k-i(x)-1}-1$.

It is easy to see that $A_{k}$ is a subtree of $\mathbf{Z}^{2}$ and that the above decomposition of $x$ is unique. In particular, we can consider its intrinsic graph metric $d_{A_{k}}$ : let $S_{k}$ be the sphere of center $a_{k}$ and of radius $2^{k+1}-1$ for this metric. Clearly, $\left|S_{k}\right| \geq 3^{k-1}$.

Second step of the construction: We define a graph $Y$ with weighted edges as follows: $Y$ is the usual Cayley graph of $\mathbf{Z}^{2}$; all edges of $Y$ have length 1 but those belonging to $A=\cup_{k} A_{k}$ which have length equal to $1 / 100$. The measure on $Y$ is the countable measure and the distance between two vertices $v$ and $w$ is the minimal length of a chain joining $v$ to $w$, the length of a chain being the sum of the weights of its edges. Clearly, as a metric measure space, $Y$ is large-scale equivalent to $\mathbf{Z}^{2}$.

For every $k \geq 2$, consider the sphere $S\left(a_{k}, r_{k}\right)=B\left(a_{k}, r_{k}+1\right) \backslash$ $B\left(a_{k}, r_{k}\right)$ of $Y$, where $r_{k}=\left(2^{k+1}-1\right) / 100$.

Claim 4.11. We have $S_{k} \subset S\left(a_{k}, r_{k}\right)$, so that

$$
\mu\left(S\left(a_{k}, r_{k}\right)\right) \geq 3^{k-1} \geq r_{k}^{\log 3 / \log 2} .
$$

Proof: Note that the claim looks almost obvious on a drawing. Nevertheless, for the sake of completeness, we give a combinatorial proof. Let us show that a geodesic chain in the tree $A_{k}$ is also a minimizing geodesic chain in $Y$. Applying this to a geodesic chain between $a_{k}$ and any element of $S_{k}$ (which is of length $r_{k}$ in $Y$ ), we have that $S_{k} \subset S\left(a_{k}, r_{k}\right)$, so we are done.

So let $x$ be a vertex of $A_{k}$. By (4.2), we have

$$
x=a_{k}+2^{k} \varepsilon_{0}(x)+2^{k-1} \varepsilon_{1}(x)+\cdots+2^{k-i(x)} \varepsilon_{i(x)}(x)+r(x) \varepsilon_{i(x)+1}(x) .
$$

Let us show by recurrence on $d_{Y}\left(a_{k}, x\right)$ (which takes discrete values) that

$$
\begin{aligned}
d_{Y}\left(a_{k}, x\right) & =d_{A_{k}}\left(a_{k}, x\right) / 100 \\
& =\left(2^{k}+\cdots+2^{k-i(x)}+r(x)\right) / 100 \\
& =\frac{2^{k+1}\left(1-2^{-i(x)-1}+r(x)\right)}{100} .
\end{aligned}
$$

If $x=a_{k}$, there is nothing to prove. Consider $c=(c(0)=x, c(2), \ldots, c(m)=$ $a_{k}$ ) a minimal geodesic chain in $Y$ between $a_{k}$ and $x$. Clearly, it suffices 
to prove that $c \subset A_{k}$. Suppose the contrary. Let $t$ be the largest positive integer such that $c(t)$ belongs to $A_{k}$ and $c(t+1)$ does not. Let $l$ be the smallest positive integer such that $c(t+l) \in A_{k}$, so that $(c(t+1), \ldots, c(t+l-1))$ is entirely outside of $A_{k}$. By recurrence, the chain $(c(t+l), \ldots, c(m))$ is in $A_{k}$. Thus we have

$d_{Y}\left(x, a_{k}\right)=d_{A_{k}}(x, c(t)) / 10+|c(t)-c(t+l)|_{\mathbf{Z}^{2}}+d_{A_{k}}\left(c(t+l), a_{k}\right) / 100$.

Since $c$ is a minimal chain, we also have

$$
d_{Y}\left(c(t), a_{k}\right)=|c(t)-c(t+l)|_{\mathbf{z}^{2}}+d_{A_{k}}\left(c(t+l), a_{k}\right) / 100 .
$$

The following lemma applied to $u=c(t)$ and $v=c(t+l)$ implies that $t=t+l$ which is a contradiction since it means that $c$ is included in $A_{k}$.

Lemma 4.12. Let $u$ and $v$ be in $A_{k}$. We have

$$
|u-v|_{\mathbf{Z}^{2}} \geq\left(d_{A_{k}}\left(u, a_{k}\right)-d_{A_{k}}\left(v, a_{k}\right)\right) / 50 .
$$

Proof: We can of course assume that $d_{A_{k}}\left(u, a_{k}\right) \geq d_{A_{k}}\left(v, a_{k}\right)$. Let $u=$ $u_{1}+u_{2}$ and $v=v_{1}+v_{2}$ with

$$
u_{1}=2^{k} \varepsilon_{0}(u)+\cdots+2^{k-i(v)} \varepsilon_{i(v)}(u)
$$

and

$$
v_{1}=2^{k} \varepsilon_{0}(v)+\cdots+2^{k-i(v)} \varepsilon_{i(v)}(v) .
$$

Note that by construction,

$$
d_{A_{k}}\left(u_{1}, a_{k}\right)=d_{A_{k}}\left(v_{1}, a_{k}\right)
$$

and since $A_{k}$ is a tree,

(4.3) $d_{A_{k}}\left(u, a_{k}\right)-d_{A_{k}}\left(v, a_{k}\right)=d_{A_{k}}\left(u_{2}, a_{k}\right)-d_{A_{k}}\left(v_{2}, a_{k}\right) \leq 2^{k-i(v)+2}$.

On the other hand, we have

$$
|u-v|_{\mathbf{Z}^{2}} \geq|| u_{1}-\left.v\right|_{\mathbf{Z}^{2}}-\left|u_{2}-v\right|_{\mathbf{Z}^{2}} \mid .
$$

First, assume that $u_{1} \neq v_{1}$. Then, by (4.2), the projection of $u_{1}-v_{1}$ along $e_{1}$ or $e_{2}$ is not zero and belongs to $2^{k-i(v)} \mathbf{N}$. Moreover, using the fact that $\left.\varepsilon_{j+1}(u) \neq-\varepsilon_{j}(u)\right)$ for every $j$, the same projection of $u_{2}-v_{2}$ is (in $\mathbf{Z}^{2}$-norm) less than

2. $\left(2^{k-i(v)-2}+2^{k-i(v)-4}+\cdots=2^{k-i(v)-1}\left(1+1 / 4+1 / 4^{2}+\cdots\right) \leq 2 / 3.2^{k-i(v)}\right.$.

Thus,

$$
|u-v|_{\mathbf{Z}^{2}} \geq 2^{k-i(v)} / 3
$$

So we are done. 
Now, assume that $u_{1}=v_{1}$. If $i(u)=i(v)$ or if $i(u) \leq i(v)+1$ and $\varepsilon_{i(v)+1}(u)= \pm \varepsilon_{i(v)+1}(v)$, then we have trivially

$$
|u-v|_{\mathbf{Z}^{2}}=\left(d_{A_{k}}\left(u, a_{k}\right)-d_{A_{k}}\left(v, a_{k}\right)\right) .
$$

Otherwise, we have

$$
\begin{aligned}
u-v= & u_{2}-v_{2} \\
= & \left(2^{k-i(v)-1}-r(v)\right) \varepsilon_{i(v)+1}(u) \\
& +2^{k-i(v)-2} \varepsilon_{i(v)+2}(u)+\cdots+r(u) \varepsilon_{i(u)+1} .
\end{aligned}
$$

So, projecting this in the direction of $\varepsilon_{i(v)+2}(u)$, and since $\varepsilon_{i(v)+3}(u) \neq$ $-\varepsilon_{i(v)+2}(u)$, we obtain

$$
\begin{aligned}
|u-v|_{\mathbf{Z}^{2}} & =\left|u_{2}-v_{2}\right|_{\mathbf{Z}^{2}} \\
& \geq 2^{k-i(v)-2}-\left(2^{k-i(v)-4}+\cdots+2^{k-i(u)}+r(u)\right) \\
& \geq 2^{k-i(v)-2}-2^{k-i(v)-3}=2^{k-i(v)-3} .
\end{aligned}
$$

Together with (4.3), we get

$$
|u-v|_{\mathbf{Z}^{2}} \geq 32\left(d_{A_{k}}\left(u, a_{k}\right)-d_{A_{k}}\left(v, a_{k}\right)\right)
$$

which proves the lemma.

Clearly, $Y$ is quasi-isometric to $\mathbf{Z}^{2}$. It is not difficult (and left to the reader) to see that we can adapt the construction to obtain a graph.

Now, let us explain briefly how we can adapt the construction to obtain a Riemannian manifold bi-Lipschitz equivalent to $\mathbf{R}^{2}$. First, we embed $\mathbf{Z}^{2}$ into $\mathbf{R}^{2}$ in the standard way, so that $A_{k}$ is now a subtree of $\mathbf{R}^{2}$. Let $\tilde{A}$ be the $1 / 100$-neighborhood of $A$ in $\mathbf{R}^{2}$. Let $f$ be a nonnegative function defined on $\mathbf{R}^{2}$ such that $1-f$ is supported by $\tilde{A}, f \geq a$ and $f(x)=a$ for all $x \in A$. Finally, define a new metric on $\mathbf{R}^{2}$ multiplying the Euclidean one by $f$.

\subsection{Strongly-(IB) implies the strong isoperimetric inequality.}

The converse to Proposition 4.5 is clearly false (see the examples of the next section). However, one has

Proposition 4.13. Let $X$ be a doubling (M)-space. Suppose moreover that there exists $x \in X$ such that the family of balls of center $x$ is strongly asymptotically isoperimetric. Then we have

$$
I_{\mathcal{B}}^{\downarrow} \succeq \operatorname{id} / \phi .
$$

In particular, $X$ satisfies a strong isoperimetric inequality. 
Proof: Since $(B(x, r))_{r}$ forms an asymptotically isoperimetric family, it is enough to show that there exists $c>0$ such that

$$
\mu(\partial B(x, r)) \geq c \frac{\mu(B(x, r))}{r} .
$$

But, let us recall that property (M) implies that there exists $C>0$ such that, for all $r>0$

$$
\mu\left(C_{r, r+1}(x)\right) \leq C \mu\left(\partial_{1} B(x, r)\right) .
$$

Since $(B(x, r))_{r}$ forms an asymptotically isoperimetric family, there exists $C^{\prime} \geq 1$, such that, for all $r^{\prime}<r$

$$
\mu\left(\partial B\left(x, r^{\prime}\right)\right) \leq C^{\prime} \mu(\partial B(x, r)) .
$$

Using these two remarks, we get

$$
\mu(B(x, r)) \leq C C^{\prime} r \mu(\partial B(x, r)) .
$$

So we are done.

\section{What can happen if the profile is not strong}

All the metric measure spaces built in this section will be graphs with polynomial growth. For simplicity, we write $|A|$ for the cardinal of a finite subset $A$ of a graph.

\subsection{Bounded profile: connected isoperimetric sets are "con- trolled" by balls.}

We will say that a subset $A$ of a metric space is metrically connected (we will merely say "connected" from now on) if there does not exist any nontrivial partition of $A=A_{1} \sqcup A_{2}$ with $d\left(A_{1}, A_{2}\right) \geq 10$.

Let $X$ be a uniformly $1 / 2$-connected space, with bounded profile, and such that the measures of balls of radius $1 / 2$ are larger than a constant $a>0$. Actually, we can ignore nonconnected sets. Indeed if $\left(A_{n}\right)$ is an isoperimetric family, then the $A_{n}$ have a bounded number of connected components: otherwise, by Proposition 1.13, the boundary of $A_{n}$ would not be bounded (because the distinct connected components have disjoint 1-boundaries each one containing a ball of radius $1 / 2$ ). It suffices to replace $A_{n}$ by its connected component of maximal volume. 
Claim 5.1. Let $(X, d, \mu)$ be a $(D V)_{\text {loc}}$, uniformly $1 / 2$-connected space such that the measure of balls of radius $1 / 2$ is more than $a>0$ and whose profile $I$ is bounded. Then, if $\left(A_{n}\right)$ is an isoperimetric sequence of connected subsets of $X$, there exist a constant $C>0$, some $x_{n} \in X$ and some $r_{n}>0$ such that

$$
\forall n, \quad B\left(x_{n}, r_{n}\right) \subset A_{n} \subset B\left(x_{n}, C r_{n}\right) .
$$

Proof: To fix ideas, let us assume that $\partial A=\partial_{1} A$ (for all $A \subset X$ ). Let $y_{n}$ be a point of $A_{n}$ and write $d_{n}=\sup _{y \in \partial A_{n}} d\left(y_{n}, y\right)$. Let $r \leq d_{n}$ be such that $C_{r, r+1}\left(y_{n}\right)$ intersects nontrivially $\partial A_{n}$ (recall that $C_{r, r^{\prime}}(x)=$ $\left.B\left(x, r^{\prime}\right) \backslash B(x, r)\right)$. Then, by Proposition 3.6, there exists a constant $C \geq 1$ such that $C_{r-C, r+C}\left(y_{n}\right) \cap \partial A_{n}$ contains a ball of radius $1 / 2$ and therefore has measure $\geq a$. Consequently, if $\delta_{n}=\sup \left\{r^{\prime}-r ; C_{r, r^{\prime}}\left(y_{n}\right) \cap \partial A_{n}=\emptyset\right\}$, then

$$
\mu\left(\partial A_{n}\right) \geq \frac{d_{n}}{2 C \delta_{n}} a
$$

Since the boundary of $A_{n}$ has bounded measure, there exists a constant $c>0$ and, for all $n$, two positive reals $r_{n}^{\prime}$ and $r "{ }_{n}$ such that $r_{n}{ }_{n}-r_{n}^{\prime} \geq c d_{n}$ and $C_{r_{n}^{\prime}, r{ }_{n}} \cap \partial A=\emptyset$.

Write $s_{n}=\left(r_{n}^{\prime}+r_{n}^{\prime \prime}\right) / 2$. Since $A_{n}$ is connected, $C_{s_{n}-10, s_{n}+10}(x) \cap A_{n}$ is nonempty. But then, if $x_{n} \in C_{s_{n}-10, s_{n}+10}(x) \cap A_{n}$, we get

$$
B\left(x_{n}, \frac{r_{n}-r_{n}^{\prime}}{2}-10\right) \subset A_{n} .
$$

On the other hand

$$
A_{n} \subset B\left(x_{n}, 2 d_{n}\right) .
$$

Write $r_{n}=c d_{n} / 2-10$. The claim follows from (5.1) and from (5.2).

5.2. Stably-(NIB) graphs with unbounded profile and where isoperimetric families can never be "controlled" by families of balls.

Theorem 5.2. For every integer $d \geq 2$, there exists a graph $X$ of polynomial growth of degree $d$, with unbounded profile, satisfying stably-(NIB) and such that, for all isoperimetric sequences $\left(A_{n}\right)$, it is impossible to find sequences of balls $B_{n}=B\left(x_{n}, r_{n}\right)$ and $B_{n}^{\prime}=B\left(x_{n}^{\prime}, r_{n}^{\prime}\right)$ of comparable radii (i.e. such that $r_{n}^{\prime} / r_{n}$ is bounded) such that

$$
B_{n} \subset A_{n} \subset B_{n}^{\prime}, \quad \forall n .
$$


Consider the graph $X$ obtained from $\mathbf{Z}^{d}$ deleting some edges. Consider, in the axis $\mathbf{Z} . e_{1}$, the intervals $\left(I_{n}\right)$ of length $[\sqrt{n}]$ and at distance $2^{n}$ from one another. Consider the sequence $\left(A_{n}\right)$ of full parallelepiped defined by the equations $x_{1} \in I_{n}$ and $\left|x_{i}\right| \leq n / 2$ for $i \geq 2$.

Then consider a partition of the boundary (in $\mathbf{Z}^{d}$ ) of $A_{n}$ in $(d-1)$-dimensional cubes $a_{n}^{k}$ whose edges have length approximatively $\sqrt{n}$. Remove all the edges that connect $A_{n}$ to its complement but those connected to the "center" of $a_{n}^{k}$ (here, the center of $a_{n}^{k}$ is a point of $\mathbf{Z}^{d}$ we choose at distance $\leq 2$ from the "true center" in $\mathbf{R}^{n}$ of the convex hull of $a_{n}^{k}$ ). We thus obtain a connected graph $X$. Note that the $A_{n}$ are such that

$$
\left|A_{n}\right| \approx n^{d-1} \sqrt{n}
$$

and

$$
\left|\partial_{X} A_{n}\right| \approx \frac{\left|\partial_{\mathbf{Z}^{d}} A_{n}\right|}{\left|a_{n}^{0}\right|} \approx n^{d-1} /(\sqrt{n})^{d-1}=(\sqrt{n})^{d-1} .
$$

Write $A$ for the union of $A_{i}$ and $A^{c}$ for its complement in $X$.

Claim 5.3. The growth in $X$ is polynomial of degree $d$.

Proof: It will follow from the strong profile of balls.

Claim 5.4. The profile of $X$ is not strong.

Proof: Let us consider the $A_{n}$. If the profile was strong, the sequence $u_{n}=\frac{\left|A_{n}\right|}{\left|\partial A_{n}\right|^{\frac{d}{d-1}}}$ would be bounded. But there exists a constant $c>0$ such that

$$
u_{n} \geq c n^{d-1} \sqrt{n} /(\sqrt{n})^{d}=c n^{\frac{d-1}{2}} \rightarrow \infty .
$$

Claim 5.5. Let $R$ be a unbounded subset of $\mathbf{R}_{+}$and let $\left(P_{r}\right)_{r \in R}$ be a family of subsets such that there exist two constants $C \geq 1$ and $a>0$ such that

$$
\forall r>0, \exists x_{r} \in X, \quad B\left(x_{r}, r / C\right) \subset\left[P_{r}\right]_{a} \subset B\left(x_{r}, C r\right) .
$$

Then there exists a constant $c^{\prime}$ such that

$$
\forall r>0, \quad \mu\left(\partial P_{r}\right) \geq c^{\prime} \mu\left(P_{r}\right)^{\frac{d-1}{d}} .
$$

The following lemma and its proof will be useful in all examples that we will expose in the following sections. Write $A^{c}$ for the complement of $A$ (in $X$ or, which is actually the same in $\mathbf{Z}^{d}$ ). 
Lemma 5.6. The profile of $A^{c}$ (or of $A^{\prime c}$ ) is strong. That means $I(t) \approx$ $t^{\frac{d-1}{d}}$.

Proof of the lemma: First of all, it is enough to consider only connected subsets $P$ of $A^{c}$. Indeed, if $P$ has many connected components $P_{1}, \ldots, P_{k}$, then, by subadditivity of the function $\phi: t \rightarrow t^{\frac{d-1}{d}}$, if the $P_{i}$ verify $\left|\partial P_{i}\right| \geq c \phi\left(\left|P_{i}\right|\right)$, then so do $P$.

Note that $A^{c}$ embeds into $X$ and into $\mathbf{Z}^{d}$. The idea consists in comparing the profile of $A^{c}$ to that of $\mathbf{Z}^{d}$. First of all, let us assume that a connected subset $P$ of $A^{c}$-seen in $X$ - intersects the boundary of many $A_{n}$. Then, as $\left|A_{n}\right|$ is negligible compared to the distance between the $A_{n}$ when $n$ goes to infinity, the set of points of $\partial_{\mathbf{Z}^{d}} P$ at distance 1 of $A$ has negligible volume compared to $|\partial P|$. Thus, if $|P|$ and $n$ are large enough, we get

$$
\left|\partial_{A^{c}} P\right| \geq \frac{1}{2}\left|\partial_{\mathbf{Z}^{d}} P\right| .
$$

So it is enough to consider subsets meeting only one $A_{n}$. But the complement of a convex polyhedra of $\mathbf{Z}^{d}$ has trivially the same profile (up to a constant) as $\mathbf{Z}^{d}$. So we are done.

Proof of the Claim 5.5: Let $\left(P_{r}\right)$ be a family of subsets of $X$ satisfying the condition of the proposition. We have to show that $\forall r,\left|\partial P_{r}\right| \geq$ $c^{\prime}\left|P_{r}\right|^{\frac{d-1}{d}}$. If $P \subset A^{c}$, the claim is a direct consequence of the lemma.

Suppose that $P$ meets some $A_{n}$ and that $r \geq 100 C \sqrt{n}$. Then we have already seen (in the proof of Lemma 5.6) that if many $A_{n}$ intersect $P_{r}$, the cardinal of the intersection of this $P_{r}$ with $A$ are negligible compared to its boundary provided $n$ and $\left|P_{r}\right|$ are large enough. We can thus suppose that $P_{r}$ meets only one $A_{n}$. Furthermore, since $r \geq 100 \sqrt{(n)}$, there is some $x^{\prime}$ in $B\left(x_{r}, r / C\right)$ such that

$$
B\left(x^{\prime}, r / 10 C\right) \in B\left(x_{r}, r / C\right) \cap A^{c} .
$$

Then, observe that since $B\left(x^{\prime}, r / 10 C\right) \subset\left[P_{r}\right]_{a}$, there is a $B\left(x^{\prime}, r / 10 C\right) \subset$ $\left[P_{r}\right]_{a}$, there is a constant $c>0$ such that

$$
\left|P_{r} \cap B\left(x^{\prime}, r / C\right)\right| \geq c\left|B\left(x^{\prime}, r / C\right)\right| .
$$

It follows that the intersection of $P_{r}$ with $A^{c}$ has volume $\geq c^{\prime}\left|P_{r}\right|$ where $\bar{c}$ is a constant depending only on $C$ and $a$. So by Lemma 5.6, we have

$$
\left|\partial_{X} P_{r}\right| \geq\left|\partial_{A^{c}}\left(P_{r} \cap A^{c}\right)\right| \geq c\left|P_{r}\right|^{\frac{d-1}{d}} .
$$

We then have to study the case $r \leq 100 C \sqrt{n}$. We can assume that $x_{r} \in A_{n}$ (otherwise, we conclude with Lemma 5.6). Let $\pi$ be the orthogonal projection on the hyperplane $x_{2}=0$. Then for $n$ large enough, 
$\mathrm{Cr}$ is smaller than $n / 2$. Consequently, since $P_{r} \in B\left(x_{r}, C r\right)$, every point of $\pi\left(P_{r}\right)$ has at least one antecedent in the boundary of $P_{r}$. So, we have

$$
\left|\partial_{X} P_{r}\right| \geq\left|\pi\left(P_{r}\right)\right| \text {. }
$$

Moreover, note that $\pi\left(B\left(x_{r}, r / C\right)\right)=B\left(\pi\left(x_{r}\right), r / C\right)$ (note that this ball lies in $\left.\mathbf{Z}^{d-1}\right)$. On the other hand, since the projection is 1-Lipschitz, we get

$$
\pi\left(\left[P_{r}\right]_{a}\right) \subset\left[\pi\left(P_{r}\right)\right]_{a},
$$

so

$$
B\left(\pi\left(x_{r}\right), r / C\right) \subset\left[\pi\left(P_{r}\right)\right]_{a} .
$$

Similarly to (5.3), we have

$$
\mid \pi\left(P_{r}\right) \cap B\left(\pi\left(x_{r}\right), r / C|\geq c| B\left(\pi\left(x_{r}\right), r / C\right) \mid .\right.
$$

So, finally, we have

$$
\left|\partial_{X} P_{r}\right| \geq c^{\prime} r^{d-1}
$$

so we are done.

Corollary 5.7. In every space isometric at infinity to $X$, the volume of spheres $\approx r^{d-1}$. In particular, they are not asymptotically isoperimetric.

Proof of the corollary: Let $f: X^{\prime} \rightarrow X$ a large-scale equivalence between two metric measure spaces $X^{\prime}$ and $X$ and take $y \in X^{\prime}$. It comes

$$
B\left(f(y), \frac{r}{C_{2}}-C_{1}\right) \subset B\left([f(B(y, r))]_{C_{1}}\right) \subset B\left(f(y), C_{2} r+C_{1}\right) .
$$

The corollary follows from Claim 5.5 and from Theorem 3.10.

\subsection{Graphs stably-(NIB) with bounded profile.}

Theorem 5.8. For any integer $d \geq 2$, one can find a graph of polynomial growth of degree $d$, with bounded profile, and which is stably-(NIB).

The construction follows the same lines as in the previous section. Consider in $\mathbf{Z}^{d}$, a sequence $\left(C_{n}\right)$ of subsets defined by

$$
C_{n}=B\left(x_{n}, n\right) \cup B\left(x_{n}^{\prime}, n\right)
$$

where $x_{n}=\left(2^{n+1}, n-\log n, 0, \ldots, 0\right)$ and $x_{n}^{\prime}=\left(2^{n+1}, \log n-n, 0, \ldots, 0\right)$.

We disconnect $C_{n}$ from the rest everywhere but in the axis Z.e. Let $Y$ be the corresponding graph. $C_{n}$ looks like a ball (of $\mathbf{Z}^{d}$ ) "constricted" at the equator. Indeed, every point of $C_{n}$ belonging to the hyperplane $\left\{x_{2}=0\right\}$ is at distance at most $\log n$ from the boundary (in $Y$ ) of $C_{n}$. This is the property that will prevent $C_{n}$ from being "deformed" into a ball. Write $C=\cup_{n} C_{n}$. 
Lemma 5.9. The graph $C^{c}$ has a strong profile.

Proof: The demonstration is essentially the same as for Lemma 5.6.

Claim 5.10. The growth in the graph $X$ is polynomial of degree $d$.

Proof: We have to show that there exists a constant $c>0$ such that, $\forall x, r,|B(x, r)| \geq c r^{d}$ (the converse inequality following from the fact that $X$ embeds in $\mathbf{Z}^{d}$ ). Thanks to Lemma 5.9, we can suppose that $B$ is included in a $C_{n_{0}}$ so that its radius is $\leq n_{0}$.

The conclusion follows then from the next trivial fact: in $\mathbf{Z}^{d}$, if $r \leq$ $n_{0}$, the volume of the intersection of a ball of radius $n_{0}$ with a ball of radius $r \leq n_{0}$ and of center belonging to the first ball is $\geq 2^{-d}|B(x, r)| \geq$ $2^{-10 d} r^{d}$. Indeed, the worst case is when $x$ is in a "corner" of the ball. So we are done.

Claim 5.11. If $Y^{\prime}$ is a (M)-space which is isometric at the infinity to $Y$, then its balls are not asymptotically isoperimetric.

Proof: The demonstration results from the following lemma and Proposition 1.13 .

Lemma 5.12. Let $\mathbf{P}$ be an asymptotically isoperimetric family of connected subsets of $X$. Then there exists a constant $C \geq 1$ such that, for all $P \in \mathbf{P}$ of measure $>C$, there exists $n$ such that $\left|P \triangle C_{n}\right| \leq C$.

Proof: Since the profile of $C^{c}$ is strong, it is clear that for $|P|$ large enough, $P \cap C^{c}$ must be bounded. We then have to show that if $\left(P_{n}\right)$ is a sequence of subsets such that for all $n, P_{n} \subset C_{n}$ and such that $\left|P_{n}\right|$ and $\left|C_{n} \backslash P_{n}\right|$ tends to infinity, then $\left|\partial P_{n}\right|$ also tends to infinity. Suppose, for instance that $\left|P_{n}\right| \leq\left|C_{n} \backslash P_{n}\right|$. But Theorem 3.10 makes clear that this problem in $\mathbf{Z}^{d}$ is equivalent to the similar problem in $\mathbf{R}^{d}$ : that is, replacing $C_{n}$ with its convex hull $\tilde{C}_{n}$ in $\mathbf{R}^{d}$. Since the $\tilde{C}_{n}$ are homothetic copies of $\tilde{C}_{1}$, by homogeneity, we only have to show that the profile $I(t)$ of $\tilde{C}_{1}$ is $\geq c t^{\frac{d-1}{d}}$ for $0<t<\left|\tilde{C}_{1}\right| / 2$, which is a known fact (see [11]).

Let us finish the demonstration of Claim 5.11. We now have to show that the sets $C_{n}$ cannot be - up to a set of bounded measure - inverse images of balls by some large-scale equivalence. So let $\left(X^{\prime}, d, \mu\right)$ be a (M)-space and let $f: X \rightarrow X^{\prime}$ be a large-scale equivalence.

Let us consider two points of $C_{n}$ of respectively maximum and minimum $x_{2}$. The distance of each of these points to $C^{c}$ is $\geq n / 2$ and yet, every 1-chain joining them must pass through $C_{n} \cap\left\{x_{2}=0\right\}$ whose points are at distance $\leq 2 \log n$ from $C^{c}$. But this is impossible for a ball in a (M)-space. Indeed, in a ball $B=B(o, R)$ with $R \geq N$, if a point $x$ 
is at distance $c N$ from the boundary, then the points belonging to a ball centered in $x$ and of radius $c N / 2$ are at distance at least $c N / 2$ from the boundary of $B$. But this ball intersects the ball centered in $o$ and of radius $R-c N / 2$. Moreover, by property $(\mathrm{M})$, there exists a 1 -chain joining $x$ to $o$ and staying in $B(o, R-c N / 2)$, so at a distance of the order of $N$ from boundary of $B$.

\subsection{The instability of (IB) under quasi-isometry between graphs of polynomial growth.}

Theorem 5.13. Let $d$ be an integer $\geq 2$. There exists two graphs $X$ and $X^{\prime}$ quasi-isometric, of polynomial growth of degree $d$ and with bounded or unbounded profile, such that $X$ satisfies (IB) but not $X^{\prime}$.

Like in the examples of the two previous sections, we will build a graph $X$ removing some edges from $\mathbf{Z}^{d}$ : for $n \in \mathbf{N}$, let $A_{n}$ be the ball of radius $n$ whose center belongs to the axis $\mathbf{Z} . e_{1}$ in such a chain that $A_{n+1}$ is at distance $2^{n}$ from $A_{n}$. We then remove all the edges of the boundary of $A_{n}$ but those belonging to the line $\mathbf{Z} . e_{1}$. We write $A$ for the union of $A_{n}$. The graph $X^{\prime}$ is obtained from $X$ by taking its image by the linear map fixing the first coordinate and acting on the orthogonal as an homothetic transformation of ratio 4 (it is clear that it is a quasiisometry). More precisely, we replace each edge of $X$ parallel to the first axis, by a chain of length 2 also parallel to the first axis. Write $A^{\prime}$ for the image of $A$.

Remark 5.14. In the previous example, the profile is bounded. Nevertheless, one can slightly modify the construction in order to get an unbounded profile: for instance, removing only edges of the boundary of $A_{n}$ at distance $\geq \log n$ from the axis Z. $e_{1}$ (instead of those which are outside of this axis).

Claim 5.15. The graphs $X$ and $X^{\prime}$ have polynomial growth of degree $d$.

As these graphs are subgraphs of $\mathbf{Z}^{d}$, their volume growths are less than the one of $\mathbf{Z}^{d}$. The converse inequality will follow from the fact that in $X^{\prime}$, the profile restricted to balls is strong and from the fact that $X$ and $X^{\prime}$ are quasi-isometric.

Claim 5.16. In $X$, the balls are asymptotically isoperimetric.

Proof: It is clear by construction that the $A_{n}$ are balls and that their boundaries have bounded volume.

Claim 5.17. In $X^{\prime}$, the profile restricted to balls is strong $I_{\mathcal{B}}^{\downarrow}(t) \approx t^{\frac{d-1}{d}}$. In particular, $X^{\prime}$ is not (IB). 
Proof: Remark that Lemma 5.6 stays true in this context. Let $B=$ $B(x, r)$ be a ball of the graph $X^{\prime}$. We have to show that there exists a constant $c>0$ such that

$$
|\partial B| \geq c|B|^{\frac{d-1}{d}} .
$$

According to Lemma 5.6, we can assume that $B \subset A$. Thus, there exists $n_{0}$ such that $B \subset A_{n_{0}}$.

Let us embed $\mathbf{Z}^{d}$ into $\mathbf{R}^{d}$. Let us replace the discrete polyhedron $A_{n}$ and $B$ by their convex hulls $\tilde{A_{n}}$ and $\tilde{B}$ in $\mathbf{R}^{d}$. Let $\tilde{X}$ be the space obtained removing from $\mathbf{R}^{d}$ (Euclidean) the points of the Euclidean boundary of $\tilde{A_{n}}$ (for all $n$ ) but the two ones belonging to the axis R. $e_{1}$ (resp. those at distance $\leq \log n$ of the axe) for the case of bounded profile (resp. for the case of unbounded profile). Let us equip $\tilde{X}$ - seen as a subset of $\mathbf{R}^{d}$ - with Lebesgue measure and with the geodesic metric $d(x, y)=\inf _{\gamma} l(\gamma)$ with $\gamma$ taking values in the set of arcs joining $x$ to $y$ in $\tilde{X}, l(\gamma)$ being the Euclidean length of $\gamma$.

The embedding $j$ of $X$ into $\tilde{X}$ we obtain like this is clearly a large-scale equivalence.

For simplicity, we will write $|A|$ for the (Lebesgue) measure of a subset $A$ of $\tilde{X}$. On the other hand, note that $\partial_{10} \tilde{B}$ contains $[j(B(x, r))]_{1} \backslash$ $[j(B(x, r-2))]_{1}$, which by Proposition 3.6 has same measure (up to multiplicative constant) as $\partial B$. The same holds for $\tilde{B}$ and $B$. Moreover, since $\tilde{B}$ and $A_{n_{0}}$ are convex polyhedra, it is clear that the 10-boundary of $\tilde{B}$ has same measure (up to multiplicative constants) as its Euclidean boundary (whose measure is the limit when $h \rightarrow 0$ of $\left|\partial_{h} \tilde{B}\right| / h$ ). Write

$$
\left|\partial_{\text {eucl }} \tilde{B}\right|=\lim _{h \rightarrow 0}\left|\partial_{h} \tilde{B}\right| / h .
$$

Consequently, it is enough to show that there exists $c>0$ such that

$$
\left|\partial_{\text {eucl }} \tilde{B}\right| \geq c|\tilde{B}|^{\frac{d-1}{d}} .
$$

Note that by homogeneity, the quantity

$$
Q=\frac{1}{r^{d-1}}\left|\partial_{\text {eucl }} \tilde{B}\right|
$$

only depends on the ratio $n / r$. Fix $n=n_{0}$. For $r$ small enough (let us say $\leq r_{c}$ for some $\left.r_{c}>0\right), \tilde{B}$ never meets two parallel faces: $Q$ stays larger than a constant $>0$ (i.e. profile of a $1 / 2^{d-1}$ ' th of space of $\mathbf{R}^{d}$ ). By compactness, it follows that $Q$ reaches its minimum when $x$ and $r$ vary under the conditions $r_{c} \leq r \leq n_{0} / 2$. On the other hand, as $\tilde{B}$ is strictly included in $\tilde{A_{n_{0}}}$, this minimum has to be $>0$. The ratio $Q$ is therefore 
larger than a constant $c^{\prime}>0$. finally, there is a constant $c>0$ such that

$$
\left|\partial_{\text {eucl }} \tilde{B}\right| \geq c^{\prime} r^{d-1} \geq c|B|^{\frac{d-1}{d}} .
$$

So we are done.

\section{Asymptotic isoperimetry of connected subsets}

Let $X$ be a metric measure space. Set $\partial A=\partial_{1} A$ and assume that $X$ is uniformly $1 / 2$-connected (see Section 3.1 ). Recall that we say that a subset $A$ of $X$ is connected if there does not exist a nontrivial partition $A=A_{1} \sqcup A_{2}$ with

$$
\partial A_{1} \cap \partial A_{2}=\emptyset .
$$

Write $\mathcal{C}$ for the set of connected subsets of finite measure of $X$.

Theorem 6.1. (i) Let $X$ be such that the measures of balls of radius $1 / 2$ are bounded below by $a>0$. Suppose that $I(t)=o(t)$. Then there exists a positive and increasing sequence $\left(t_{n}\right)$ tending to infinity such that $I_{\mathcal{C}}^{\downarrow}\left(t_{n}\right)=I\left(t_{n}\right)$.

(ii) Assume that $X$ is a doubling $(M)$-space and has a strong profile. Then $I_{\mathcal{C}}^{\downarrow} \approx I$.

(iii) Let $d$ be an integer $\geq 2$. There exists a graph $X$ of polynomial growth of degree $d$ and a increasing sequence of integers $\left(s_{n}\right)$ such that $I\left(s_{n}\right)=o\left(I_{\mathcal{C}}^{\downarrow}\left(s_{n}\right)\right)$.

Proof: Note that (ii) follows from Corollary 4.5 and from the fact that property (M) implies that balls are connected.

Let us show the first assertion of the theorem. Suppose that there exists $T>0$ such that $\forall t \geq T, I(t)<I_{\mathcal{C}}^{\downarrow}(t)$. We will show that it implies that

$$
I(t) \geq a \frac{t}{T}
$$

Write $t_{m}$ for the upper bound of the set of $t$ such that $\forall s \leq t$, one has $I(s) \geq a \frac{s}{T}$. Since $I$ is nondecreasing, if $t_{m}$ is finite, then it is a maximum.

Remark that $t_{m} \geq T$ since the boundary of every nonempty subset of $X$ contains a ball of radius 1/2 (see Proposition 3.6) and therefore has measure $\geq a$.

Suppose by contradiction that $t_{m}$ is finite. By definition of $t_{m}$, for all $s>t_{m}$ there exists a subset $A$ such that

$$
\mu(A) \geq s
$$


and

$$
\mu(\partial A)<a s / T \text {. }
$$

Moreover, since $t_{m} \geq T$, we can suppose that

$$
\mu(\partial A)<I_{\mathcal{C}}^{\downarrow}(s)
$$

(in particular, $A$ is not connected).

It follows that there exists a smallest positive integer $k$ such that there exist $t_{m} \leq s \leq t_{m}+T / 2$ and a subset $A$ of measure $\geq s$, with $k$ connected components and whose boundary has measure $<\min \left\{I_{\mathcal{C}}^{\downarrow}(s), s a / T\right\}$. Let $A$ be such a subset. Note that $k \geq 2$. Thus, we have

$$
A=A_{1} \sqcup A_{2}
$$

with $d\left(A_{1}, A_{2}\right) \geq 10$.

Since $k$ is minimal, one has, for $i=1,2$

$$
\mu\left(A_{i}\right)<t_{m} .
$$

Indeed, if for instance, one has $\mu\left(A_{1}\right) \geq t_{m}$, then since the boundary of $A_{2}$ has measure $\geq a$, one would have

$$
\mu\left(\partial A_{1}\right) \leq\left(t_{m}+T / 2\right) \frac{a}{T}-a=\frac{t_{m} a}{T}-a / 2<\frac{t_{m} a}{T} .
$$

Therefore, as $I_{\mathcal{C}}^{\downarrow}\left(t_{m}\right) \geq I\left(t_{m}\right) \geq t_{m} a / T$, one would also have

$$
\mu\left(\partial A_{1}\right)<I_{\mathcal{C}}^{\downarrow}\left(t_{m}\right) .
$$

But then, by minimality of $k, A_{1}$ should have at least $k$ connected components, which is a contradiction since it has strictly less components than $A$.

But, by definition of $t_{m}$, this implies that

$$
\begin{aligned}
\mu(\partial A) & =\mu\left(\partial A_{1}\right)+\mu\left(\partial A_{2}\right) \\
& \geq \frac{\mu\left(A_{1}\right) a}{T}+\frac{\mu\left(A_{2}\right) a}{T} \\
& =\frac{\mu(A) a}{T}
\end{aligned}
$$

which is a contradiction.

In order to show the second assertion of the theorem, we proceed as in the previous sections: we start from the graph $\mathbf{Z}^{d}$, and then we remove some edges. Let us consider the following family of cubes $\left(C_{n}^{m}\right)_{0 \leq m \leq n-1, n \in \mathbf{N}^{*}}$ of $\mathbf{Z}^{d}$ : the $C_{n}^{m}$ are Euclidean cubes of edges' length $2^{2^{n}}$ whose centers are disposed along the axis Z $\mathbf{Z} \cdot e_{1}$ as follows: $C_{n}^{m+1}$ is the image of $C_{n}^{m}$ by the translation of vector $n 2^{2^{n}} \cdot e_{1}$ and $C_{n}^{n-1}$ 
and $C_{n+1}^{1}$ are at distance $(n+1) 2^{2^{(n+1)}}$ to one another. To build the graph $X$, we remove all the edges joining $C_{n}^{m}$ to the rest of the graph but those which have a vertex belonging to the Euclidean cube $c_{n}^{m}$ of dimension $d-1$ of the boundary of $C_{n}^{m}$, of volume $2^{n^{2}}$ and centered in one of the two intersection points of $C_{n}^{m}$ with the axis Z. $e_{1}$. Write $C$ for the union of cubes $C_{n}^{m}$.

Claim 6.2. The growth in $X$ is polynomial of degree $d$.

Proof: Let $B=B(x, r)$ be a ball. Let us prove that $|B| \geq 2^{-100 d} r^{d}$. If the center of $B$ doesn't belong to any $C_{n}^{m}$, it is clear. Suppose therefore that $x \in C_{n_{0}}^{m_{0}}$ for integers $n_{0}$ and $m_{0}<n_{0}$. Write $D_{n_{0}}$ for the diameter of $C_{n_{0}}^{m_{0}}$. If $r \geq 3 D_{n_{0}}$, then $B$ contains $B(y, r / 2)$ with $y$ belonging to no $C_{n}^{m}$. So we are brought back to the previous case. In the other case, the conclusion follows from the following trivial fact: in $\mathbf{Z}^{d}$, if $r \leq n$, the volume of the intersection of a cube $C$ of edges' length equal to $n$ with a ball of radius $r \leq n$ and of center $x \in C$ is $\geq 2^{-d}|B(x, r)| \geq 2^{-10 d} r^{d}$. Indeed, the worst case is when $x$ is a corner of the cube.

Claim 6.3. Take $s_{n}=n 2^{2^{n}}$. Then $I\left(s_{n}\right)=o\left(I_{\mathcal{C}}^{\downarrow}\left(s_{n}\right)\right)$.

Proof: Let us consider the set $C_{n}=\cup_{m} C_{n}^{m}$. Its volume is equal to $s_{n}$ and its boundary has volume equal $n 2^{n^{2}}$. On the other hand, let $n_{1}$ be an integer and let $P$ be a connected subset of volume $\geq N_{n_{1}}$. We want to show that $|\partial P| \geq c 2^{\left(n_{1}+1\right)^{2}}$, for a constant $c>0$, which is clearly enough to conclude.

Thanks to the following lemma, the only remaining case to consider is when $P$ meets a cube $C_{n}^{m}$. But, because of the large distance between two such cubes, we can assume that $P$ meets only one of these cubes, say $C_{n_{0}}^{m_{0}}$.

Lemma 6.4. The profile of the graph $C^{c}$ is strong (i.e. $\approx t^{\frac{d-1}{d}}$ ).

(Same demonstration as for Lemma 5.6.)

If $\left|P \cap C^{c}\right| \geq|P| / 2$, then the lemma applied to $P \cap C^{c}$ allows to conclude. Suppose therefore that $|P \cap C| \geq|P| / 2$. This implies in particular that $n_{0} \geq n_{1}+1$. We then remark that $\left|\partial\left(P \cap C_{n_{0}}^{m_{0}}\right)\right| \leq$ $|\partial P|$. Indeed, let $\pi$ be the orthogonal projection onto the hyperplane containing $c_{n_{0}}^{m_{0}}$, then every point of $c_{n_{0}}^{m_{0}} \cap P$ admits un antecedent by $\pi$ belonging to the boundary of $P$. So we can assume that $P \subset C_{n_{0}}^{m_{0}}$. If $|P| \leq 3 / 2\left|C_{n_{0}}^{m_{0}}\right|$, then there exists $c>0$ such that

$$
|\partial P| \geq c|P|^{\frac{d-1}{d}}
$$


(isoperimetry in the full Euclidean cube: see [11]). Otherwise, assume that $|P| \geq 3 / 2\left|C_{n_{0}}^{m_{0}}\right|$ and write $Q=C_{n_{0}}^{m_{0}} \backslash P$.

- If the volume of $Q$ is $\geq D_{n_{0}} / 2$ where $D_{n_{0}}$ is the diameter of $C_{n_{0}}^{m_{0}}$, then (6.2) applied to $Q$ implies that

$$
|\partial Q| \geq c 2^{(d-1) 2^{n_{0}} / d} \geq c 2^{2^{n_{0}-1}} \geq c 2^{n_{0}^{2}}=c 2^{\left(n_{1}+1\right)^{2}} .
$$

But, the boundary of $Q$ is - up to points belonging to $c_{n_{0}}^{m_{0}}$ (whose cardinal is negligible compared to $c 2^{2^{n_{0}-1}}$ ) - equal to the boundary volume of $P$. So we are done.

- If $|Q| \leq D_{n_{0}} / 2$, then every point of $c_{n_{0}}^{m_{0}}$ has preimages in $\partial P$ by the projector $\pi$. But $\left|c_{n_{0}}^{m_{0}}\right|=2^{n_{0}^{2}}=2^{\left(n_{1}+1\right)^{2}}$, which ends the demonstration.

\section{References}

[1] T. Coulhon and L. Saloff-Coste, Variétés riemanniennes isométriques à l'infini, Rev. Mat. Iberoamericana 11(3) (1995), 687-726.

[2] T. Coulhon and L. Saloff-Coste, Isopérimétrie pour les groupes et les variétés, Rev. Mat. Iberoamericana 9(2) (1993), 293-314.

[3] A. ERschler, On isoperimetric profiles of finitely generated groups, Geom. Dedicata 100 (2003), 157-171.

[4] K. Falconer, "Fractal geometry. Mathematical foundations and applications", John Wiley \& Sons, Ltd., Chichester, 1990.

[5] M. Gromov, Groups of polynomial growth and expanding maps, Inst. Hautes Études Sci. Publ. Math. 53 (1981), 53-73.

[6] M. Gromov, Infinite groups as geometric objects, in: "Proceedings of the International Congress of Mathematicians", Vol. 1, 2 (Warsaw, 1983), PWN, Warsaw, 1984, pp. 385-392.

[7] M. Gromov, Asymptotic invariants of infinite groups, in: "Geometric group theory", Vol. 2 (Sussex, 1991), London Math. Soc. Lecture Note Ser. 182, Cambridge Univ. Press, Cambridge, 1993, pp. 1-295.

[8] M. KanAI, Rough isometries, and combinatorial approximations of geometries of noncompact Riemannian manifolds, J. Math. Soc. Japan 37(3) (1985), 391-413.

[9] Ch. Pittet, The isoperimetric profile of homogeneous Riemannian manifolds, J. Differential Geom. 54(2) (2000), 255-302. 
[10] Ch. Pittet and L. Saloff-Coste, A survey on the relationships between volume growth, isoperimetry, and the behaviour of simple random walk on Cayley graphs, with examples, Unpublished manuscript (1997).

[11] A. Ros, The isoperimetric problem, Lecture series given during the Clay Mathematics Institute Summer School on the Global Theory of Minimal Surfaces, MSRI, Berkeley, California (2001).

[12] L. SAloff-Coste, Analysis on Riemannian co-compact covers, in: "Surveys in differential geometry", Vol. IX, Int. Press, Somerville, MA, 2004, pp. 351-384.

[13] R. S. Strichartz, Isoperimetric estimates on Sierpinski gasket type fractals, Trans. Amer. Math. Soc. 351(5) (1999), 1705-1752.

[14] R. Tessera, Asymptotic volume of spheres in metric measure spaces and in groups of polynomial growth, and its applications in ergodic theory, Preprint (2004).

[15] N. Th. Varopoulos, Analysis on Lie groups, J. Funct. Anal. 76(2) (1988), 346-410.

Département de Mathématiques

Université de Cergy-Pontoise

Site de Saint Martin

2, avenue Adolphe Chauvin

95302 Cergy-Pontoise Cedex

France

E-mail address: tessera@clipper.ens.fr

Primera versió rebuda el 5 de juliiol de 2005, darrera versió rebuda el 3 de novembre de 2005. 